\title{
Antigenicity of a whole parasite vaccine as promising candidate against canine leishmaniasis
}

\author{
Rodolfo Cordeiro Giunchetti ${ }^{\mathrm{a}, \mathrm{b}, *}$, Alexandre Barbosa Reis ${ }^{\mathrm{a}, \mathrm{b}}$, Denise da Silveira-Lemos ${ }^{\mathrm{b}}$, \\ Olindo Assis Martins-Filho ${ }^{\mathrm{c}}$, Rodrigo Corrêa-Oliveira ${ }^{\mathrm{b}}$, \\ Jeffrey Bethony ${ }^{\mathrm{b}}$, André Macedo Vale ${ }^{\mathrm{d}}$, Josiane da Silva Quetz ${ }^{\mathrm{d}}$, \\ Lilian Lacerda Bueno e, João Carlos França-Silva ${ }^{\mathrm{d}}$, Evaldo Nascimento ${ }^{\mathrm{d}}$, \\ Wilson Mayrink ${ }^{\mathrm{d}}$, Ricardo Toshio Fujiwara ${ }^{\text {b,d,* }}$ \\ ${ }^{\text {a }}$ Laboratório de Imunopatologia, Núcleo de Pesquisas em Ciências Biológicas, Universidade Federal de Ouro Preto, Brazil \\ ${ }^{\mathrm{b}}$ Laboratório de Imunologia Celular e Molecular, Centro de Pesquisas René RachoulFIOCRUZ, Avenida Augusto de Lima, \\ 1715, Belo Horizonte, Minas Gerais, CEP 30190-002, Brazil \\ c Laboratório de Biomarcadores de Diagnóstico e Monitoração, Centro de Pesquisas René RachoulFIOCRUZ, Brazil \\ ${ }^{\mathrm{d}}$ Laboratório de Leishmanioses, Universidade Federal de Minas Gerais, Brazil \\ ${ }^{\mathrm{e}}$ Laboratório de Malária, Universidade Federal de Minas Gerais, Brazil
}

Accepted 6 September 2007

\begin{abstract}
Human visceral leishmaniasis, one of the most important zoonoses, is caused by the protozoa Leishmania chagasi (syn. L. infantum) and is present as a fatal disease common in South America and Europe where dogs and wild canids are the main reservoirs. A vaccine against visceral leishmaniasis would be an important tool in the control of this disease in dogs. Although the current strategies for vaccination against leishmaniasis are based on the use of recombinant antigens, killed vaccines are still attractive in terms of stability of their biochemical composition and antigenicity, cost, and safety. Here we evaluate the immunogenicity of a whole parasite vaccine as a promising candidate against canine leishmaniasis, demonstrated by cellular reactivity, changes in the cellular profile of the peripheral blood and by the differential production of immunoglobulins. Our results showed that immunization elicited mainly a strong cellular reactivity and increase in T-lymphocytes, particularly the subpopulation CD8 ${ }^{+}$that would be related to the control of tissue parasitism. In addition, a higher production of anti-Leishmania total $\mathrm{IgG}$, characterized by mixed isotypes profile (IgG1 and IgG2), was demonstrated. (C) 2007 Elsevier Ltd. All rights reserved.
\end{abstract}

Keywords: Vaccine; Canine visceral leishmaniasis; Whole parasite vaccine; Dog

\section{Introduction}

Human visceral leishmaniasis (HVL), one of the most important zoonoses, is caused by the protozoa Leish-

\footnotetext{
* Corresponding authors. Present address: Laboratório de Imunologia Celular e Molecular, Centro de Pesquisas René Rachou/FIOCRUZ, Avenida Augusto de Lima, 1715, Belo Horizonte, Minas Gerais, CEP 30190-002, Brazil. Tel.: +55 313349 7775; fax: +55 3132953115.

E-mail addresses: giunchetti@nupeb.ufop.br (R.C. Giunchetti), fujiwara@cpqrr.fiocruz.br (R.T. Fujiwara).
}

mania (Leishmania) chagasi (syn. Leishmania. infantum) and is present as a fatal disease common in South America and Europe where dogs and wild canids are the main reservoirs (Deane and Deane, 1962). In Brazil, the prevalence for CVL in other endemic areas has shown to vary from 5\% to 35\% (Evans et al., 1990; Nunes et al., 1991).

According to Hommel et al. (1995) a vaccine against visceral leishmaniasis would be an important tool in the control of CVL, and would also dramatically decrease the pressure of infection of $L$. chagasi/L. infantum over 
humans. However, an effective vaccine against kala azar is not yet available and only few reports in the literature deal with a vaccine against canine visceral leishmaniasis (Mayrink et al., 1996; Lasri et al., 1999; Panaro et al., 2001; Silva et al., 2001; Borja-Cabrera et al., 2002; Molano et al., 2003; Fujiwara et al., 2005b; Lemesre et al., 2005; Giunchetti et al., 2007c). Although the current strategy for vaccination against leishmaniasis is based on the use of recombinant antigens, whole parasite vaccines are still attractive in terms of stability of their biochemical composition and antigenicity, cost, safety and have been used in vaccine trials against leishmaniasis (Ravindran and Ali, 2004). In dogs, these vaccines demonstrated up to $90 \%$ of protection in Phases I and II clinical trials in Brazil, using merthyolated sound-disrupted promastigotes of L. braziliensis formulated BCG (Bacillus Calmete Guérin) (Mayrink et al., 1996). The use of these whole parasite vaccines was usually accompanied by strong cellular reactivity, as observed by lymphocyte proliferation (Mayrink et al., 1996). Indeed, Lasri et al. (1999) demonstrated the vaccination of dogs with autoclaved $L$. major promastigotes (ALM) and BCG as adjuvant elicited a strong cell proliferation to leishmanial antigens compared to the others groups. Cellular and humoral immune responses of dogs to a candidate vaccine, composed of L. braziliensis promastigote protein plus saponin as an adjuvant, presented strong antigenicity related to the increases of anti-Leishmania $\mathrm{IgG}$ isotypes, higher levels of $L$. chagasi antigen-specific $\mathrm{CD}^{+} \mathrm{T}-\mathrm{lym}-$ phocytes as well as intense cell proliferation and increased nitric oxide production after in vitro stimulation by $L$. chagasi soluble antigens (Giunchetti et al., 2007c). These studies related promising results in crude antigens vaccines (Mayrink et al., 1996; Lasri et al., 1999; Panaro et al., 2001; Giunchetti et al., 2007c). Considering the facilities to manufacture crude antigens vaccines, the lower production cost and the promising results, this approach might prove to be useful in controlling leishmaniasis in endemic areas, particularly under developed countries.

Here we evaluate the immunogenicity of a whole parasite vaccine as a promising candidate against canine leishmaniasis, demonstrated by cellular reactivity and changes in the cellular profile of the peripheral blood and by the differential production of specific immunoglobulins.

\section{Materials and methods}

\subsection{Animals and vaccine study design}

Two groups of dogs were used in this study (whole parasite vaccine - WPV - and Placebo groups). The dogs were parasite naive and were selected from a colony of beagles, bred and maintained under conditions designed to exclude any possible contaminating Leishmania infections. The dogs were between 8 and 12 months old, well-fed animals under constant supervision by a veterinarian and had all received their routine vaccinations against parvovirosis, distemper, adenovirosis-2, hepatitis, parainfluenza and lep- tospirosis (Recombitek ${ }^{\circledR}$, Merial Inc., USA). All animals also received a single intranasal dose against adenovirosis-2, parainfluenza and Bordetella bronchiseptica (Bronch Shield III ${ }^{\circledR}$, Fort Dodge, USA). The dogs were also treated with anti-helminthic drugs (Endal Plus ${ }^{\circledR}$, Schering Plough, Brazil) and with anti-ectoparasites (Frontline ${ }^{\circledR}$, Merial Inc., USA), and were quarantined for approximately four weeks before beginning the vaccine trial. The animals were divided into two groups. One group, namely WPV $(n=5)$, received a whole parasite vaccine (mix of Leishmania amazonensis - IFLA/BR/1967/PH8 - and Leishmania braziliensis - MCAN/BR/1972/C348 - crude extracts) formulated with nonlive lyophilized BCG (FAP, Rio de Janeiro, Brazil) as the adjuvant. The second group, Placebo $(n=5)$, received $\mathrm{BCG}$ alone (three decreasing doses $-400 \mu \mathrm{g}$ of BCG in the first dose, $300 \mu \mathrm{g}$ in the second and $200 \mu \mathrm{g}$ in the last dose). All vaccines were administered subcutaneously in three immunizations at intervals of four weeks. Peripheral blood samples from the jugular vein of the animals were taken before the immunizations (time 0 ), 15 days after each dose of immunization (time 15: 15 days after first immunization or time 1; time 45: 15 days after second immunization or time 2; time 75: 15 days after third immunization or time 3 ) for the immune evaluations. This work was approved by the Ethical Committee of Animal Research of the Federal University of Minas Gerais, Belo Horizonte, Brazil (Protocol No. 008/02).

\subsection{Cell proliferation}

Lymphoproliferation assay was performed using $20 \mathrm{ml}$ of heparinized blood, which was collected and added on $10 \mathrm{ml}$ of the Ficoll-Hypaque density gradient (Histopaque 1.077 - Sigma Co., EUA) followed by centrifugation at $450 \mathrm{~g}$, for $40 \mathrm{~min}$, at room temperature (RT). Peripheral blood mononuclear cells (PBMC) were separated from blood, resuspended in RPMI 1640 medium (GIBCO, Grand Island, NY, USA), homogenized and washed twice in RPMI 1640 medium under centrifugation at $450 \mathrm{~g}$, for $10 \mathrm{~min}$, at RT. The PBMC was homogenized and resuspended in $10^{7}$ cells/ml in RPMI 1640. The lymphoproliferation assay was performed in 96 well flat bottom tissue culture plates (Nunc, Denmark) in $150 \mu \mathrm{l}$ with RPMI medium (supplemented with streptomycin $(100 \mathrm{mg} / \mathrm{ml})$, penicillin $(100 \mathrm{U} / \mathrm{ml})$, L-glutamine $(2 \mathrm{mM}), \beta$-mercaptoethanol $\left(5 \times 10^{-5} \mathrm{M}\right)$ and $10 \%$ heat-inactivated FCS $)$. The cells were added to triplicate wells using $25 \mu \mathrm{l}$ of PBMC $\left(2.5 \times 10^{5}\right.$ cells/well $)$ with $25 \mu \mathrm{l}$ of $L$. chagasi antigen $(10 \mu \mathrm{g} / \mathrm{ml})$ obtained according to Reis et al. (2006a,c) and $15 \mu \mathrm{l}$ of PBMC $\left(1.5 \times 10^{5}\right.$ cells/well $)$ for the mitogenic stimulus with $25 \mu$ of ConA (Concanavalin A - Sigma Chemical Co., EUA; $2 \mu \mathrm{l} /$ well). Both stimuli were compared to unstimulated control with $25 \mu$ of the RPMI 1640 medium plus $25 \mu \mathrm{l}$ of $\mathrm{PBMC}$ with $2.5 \times 10^{5}$ cells/well. Incubation was carried out in a humidified $5 \% \mathrm{CO}_{2}$ atmosphere at $37{ }^{\circ} \mathrm{C}$ for three (ConA-stimulated cultures) and five (L. chagasi-stimulated cultures) days. Six hours prior to 
termination of culture, $1 \mu \mathrm{Ci}$ of $3 \mathrm{H}$-thymidine (Sigma Chemical Co., USA) was added to each well and harvested onto glass fibre filters. Radioactive incorporation was determined by liquid scintillation spectrometry. Proliferation responses were expressed as mean counts of triplicate wells per minute and performed stimulation delta ( $\mathrm{SD}=$ mean proliferation of stimulated cultures by $L$. chagasi antigen - mean proliferation of unstimulated cultures).

\subsection{Immunophenotyping}

Immunofluorescence assay was performed according to Fujiwara et al. (2005a) and Reis et al. (2005). In brief, peripheral blood $(5 \mathrm{ml})$ was collected into EDTA containing tubes and then lysed with FACS lysing solution (FACS lysing solution - FLS - Becton Dickinson, Moutain View, CA). Cell suspension was incubated with monoclonal antibodies $(\mathrm{mAb})$ rat anti-canine Thy-1 (Rat-IgG2b: clone YKIX337.217), CD4 (Rat-IgG2a: clone YKIX302.9), CD8 (Rat-IgG1: clone YCATE55.9), fluorescein isothiocyanate conjugated (FITC) CD21 to human B lymphocytes and CD45RA (Rat-IgG2b: clone YKIX753.22.2). Polyclonal anti-rat IgG FITC conjugated was used as secondary antibody to develop the non-fluorescent antibodies. The cells were washed with PBS and analyzed on a FACScalibur flow cytometer (Becton-Dickinson, San Jose, CA, USA) using the CellQuest ${ }^{\top \mathrm{M}}$ software (Becton Dickinson, USA). Ten thousand ungated events were collected from each sample. Gating was based on forward- and side-scatter light characteristics. The results for Thy-1 ${ }^{+}$, $\mathrm{CD} 4^{+}, \mathrm{CD} 8^{+}$, and $\mathrm{CD} 21^{+}$were expressed as percentual values of cells to each marker represented by the percentage of positive lymphocytes to each surface marker staining. Semi-quantitative analyses of cell surface markers presenting unimodal distribution (CD45RA) were also stable for immunophenotyping after fixation, with no changes on fluorescence intensity distribution as determined by mean fluorescence channel (MFC). Unlabeled mAbs and polyclonal anti-rat IgG FITC conjugated used as secondary antibody in this study were purchased from SEROTEC (Oxford, UK) and anti-CD21 was purchased from Immunotech Co. (Marselle, France).

\subsection{Humoral immune response}

Antibodies were determined by conventional enzymelinked immunosorbent assay (ELISA) for the immunogenicity evaluation with soluble lysate of both $L$. amazonensis (MHOM/BR/1960/BH6) (SLaA) and L. chagasi (MHOM/ $\mathrm{BR} / 1972 / \mathrm{BH} 46$ ) (SLcA) used according to Reis et al. $(2006 \mathrm{a}, \mathrm{c})$. The antigens were coated onto 96-well microplates (Maxi-Sorp ${ }^{\mathrm{TM}}$, Nalge Nunc Intl., USA) at a concentration of $10 \mu \mathrm{g} / \mathrm{well}$ for SLcA. The sera were added at a dilution of 1:80 followed by washes and addition of peroxidase conjugated goat anti-dog IgG1 or sheep anti-dog IgG and IgG2 (Bethyl Laboratories Inc., Montgomery, TX,
USA). The wells were then washed and substrate and chromogen $(O$-Phenylenediamine, Sigma-Aldrich Co., USA) were added and the absorbance was read on an automatic ELISA microplate reader (Multiskan ${ }^{\circledR}$ MCC 340, Labsystems, Helsinki, Finland) at $492 \mathrm{~nm}$. The conjugate antiIgG1 was used at a dilution of 1:1000 and the conjugates anti-IgG and $\operatorname{IgG} 2$ were used at 1:8000 and 1:16000 dilutions, respectively.

\subsection{Statistical analysis}

Statistical analysis was performed using the Prism 3.0 software package (Prism Software, Irvine, CA, USA). The normality test Kolmogorov-Smirnoff was performed and it demonstrated the normality of the data. The repeated measures one-way analysis of variance (ANOVA) and Tukey post test were used for the longitudinal study among the different times tested in both WPV and placebo groups. Student's $t$-test was used for determining the differences between the WPV and placebo groups. The differences were considered significant when the probabilities of equality, $P$ values, were $<0.05$.

\section{Results}

\subsection{Marked increase in in vitro cell reactivity, mainly after first immunization is a hallmark of WPV}

In order to evaluate cell proliferation in the presence of the $L$. chagasi antigen as a potential vaccine against etiological agent of visceral leishmaniasis, cell reactivity was analyzed (Fig. 1). WPV showed significant improvement $(P<0.05)$ in stimulation delta $(\mathrm{SD})$ after first and second immunizations. Longitudinal analyses considering

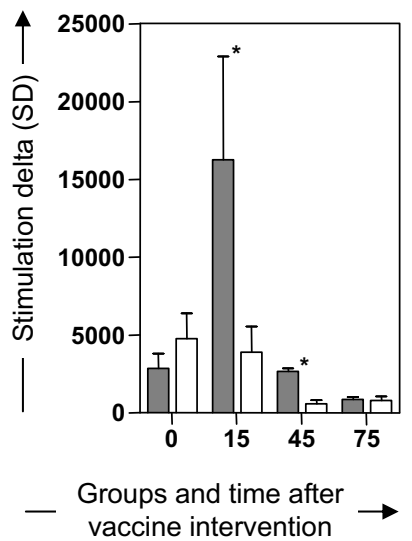

Fig. 1. Specific cell proliferation response in whole parasite vaccine (WPV - grey bars) and Placebo (white bars) from peripheral blood mononuclear cells after stimulation with soluble $L$. chagasi antigen. Results are expressed as stimulation delta (SD) and the bars represent the mean for each group plus standard deviation. The $x$-axis represents the subcutaneous doses at different times ( 0 : before the first dose; 15 : 15 days after the first dose on day 15; 45: 15 days after the second dose on day 45; 75: 15 days after the third dose on day 75). *Significant differences at $P<0.05$ are indicated in comparison to WPV and Placebo groups. 
immunizations for WPV revealed significant increase $(P<0.05)$ only in time 1 compared to time 3 .

\subsection{Enhanced frequency in Thy-1 $1^{+}$T-lymphocytes, mainly} $C D 8^{+} T$-cells as well as a decrease in $C D 4^{+} T$-cells, CD2 $1^{+}$ $B$-cells and expression in lymphocytes $C D 45 R A$ represent the major immunophenotic features in WPV

Immunophenotypic profile of peripheral blood leukocytes is presented in Fig. 2. The evaluation for T-lymphocytes and subsets $\left(\mathrm{Thy}-1^{+}, \mathrm{CD} 4^{+}\right.$and $\left.\mathrm{CD} 8^{+}\right)$showed a distinct profile. We observed that animals immunized with WPV presented a significant increase $(P<0.05)$ in T-lymphocytes Thy- $1^{+}$after second immunization compared to the Placebo group (Fig. 2a). In this context, the main subset that contributes to the augmentation of T-lymphocytes Thy $-1^{+}$was the $\mathrm{CD} 8^{+}$subpopulation that presented significant increase $(P<0.05)$ after first and second immunizations (Fig. 2c). Interestingly, when T-lymphocytes $\mathrm{CD} 4^{+}$ were evaluated in WPV, we observed a significant $(P<0.05)$ reduction after third immunization (Fig. 2b). Similarly, the evaluation in B-lymphocytes CD $21^{+}$showed a significant decrease $(P<0.05)$ in dogs that received WPV after the last dose of vaccine (Fig. 2d).
Expression of lymphocytes CD45RA by peripheral blood lymphocytes was evaluated through semi-quantitative analyses in order to evaluate whether immunizations may be associated with a distinct pattern of these constitutive cell surface markers involved directly in lymphocyte activation. Significant reduction $(P<0.05)$ in the expression of lymphocytes CD45RA after first immunization was observed in WPV (Fig. 2e). Moreover, the expression of lymphocytes CD45RA in the WPV group presented a significant decrease $(P<0.05)$ along the subsequent experimental times.

\subsection{Despite the high levels of total IgG anti-Leishmania, $W P V$ demonstrates a mixed IgG1/IgG2 response}

The reactivity of seric anti-Leishmania immunoglobulins is shown in Fig. 3. Our results demonstrated that both antiL. amazonensis (Fig. 3a) and anti-L. chagasi (Fig. 3b) total IgG presented increase after second and third immunizations in WPV. Furthermore, WPV elicited higher levels $(P<0.05)$ of anti-Leishmania IgG1 after second and third immunizations (Fig. 3c). Moreover, the longitudinal evaluation of anti- $L$. chagasi/L. amazonensis and anti-Leishmania $\operatorname{IgG} 1$ revealed that only those dogs immunized a

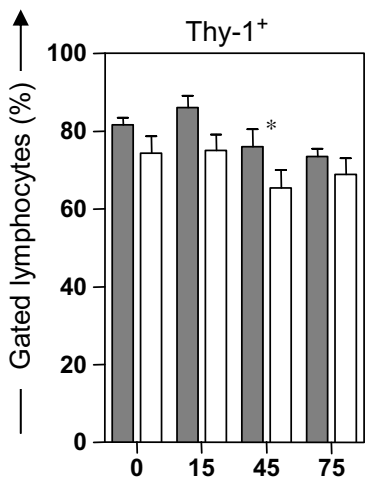

b

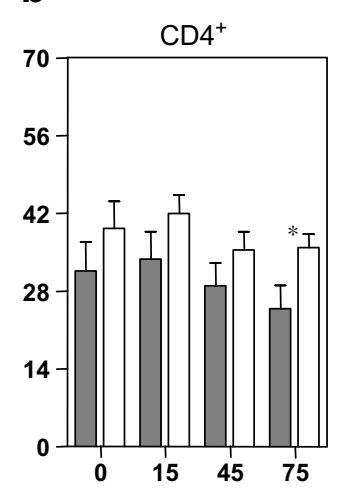

C

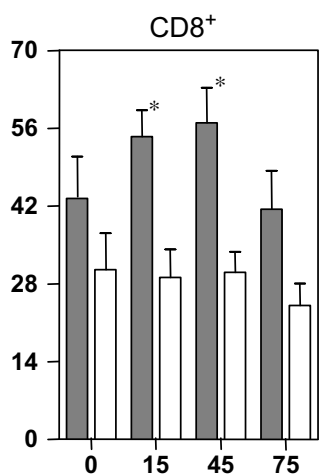

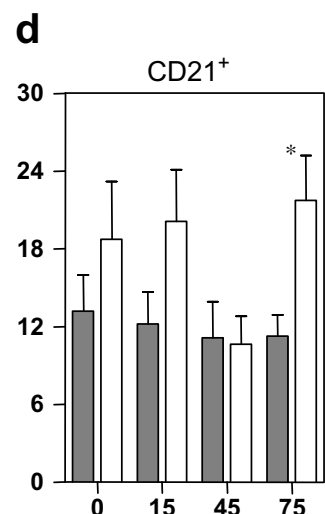

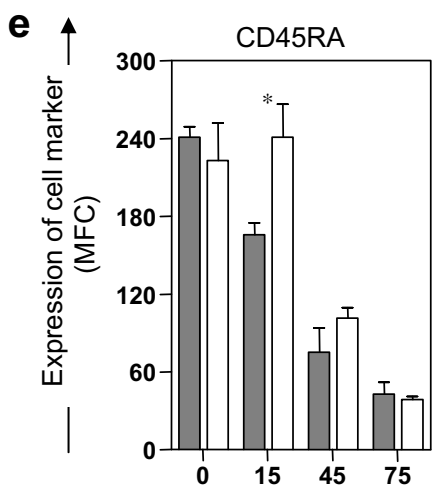

Groups and time after vaccine intervention

Fig. 2. Immunophenotypic profile of peripheral blood leukocytes in beagles that received whole parasite vaccine (WPV - grey bars) and Placebo (white bars). Results are expressed as percentage cell counts of Thy- $1^{+}(\mathrm{a}), \mathrm{CD}^{+}$(b), $\mathrm{CD} 8^{+}$(c), and $\mathrm{CD} 21^{+}$(d) lymphocytes. The results are expressed as average of CD45RA expression (e) reported as mean fluorescence channel (MFC) plus standard deviation. The $x$-axis represents the subcutaneous doses at different times ( 0 : before the first dose; 15: 15 days after the first dose on day 15; 45: 15 days after the second dose on day 45; 75: 15 days after the third dose on day 75). *Significant differences at $P<0.05$ are indicated in comparison to WPV and Placebo groups. 

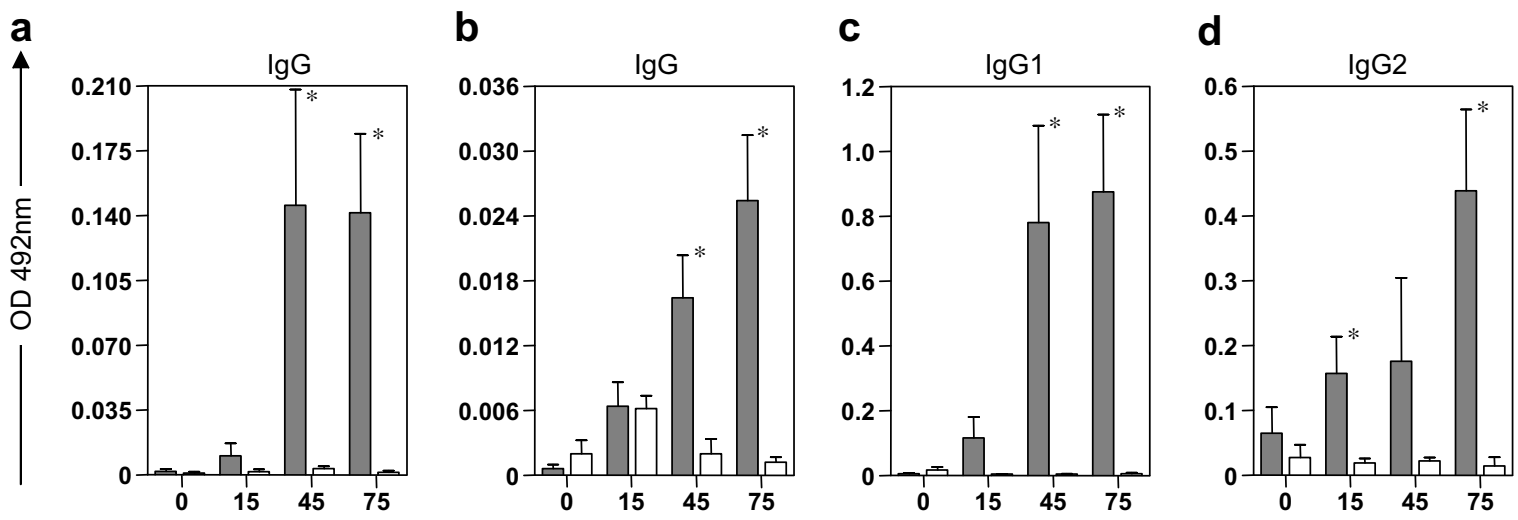

Groups and time after vaccine intervention

Fig. 3. Comparative levels of serum specific antibody production between whole parasite vaccine (WPV - grey bars) and Placebo (white bars). The results are expressed as the mean average plus standard deviation of absorbance in sera from each group. The $x$-axis represents the subcutaneous doses at different times ( 0 : before the first dose; 15: 15 days after the first dose on day 15; 45: 15 days after the second dose on day 45; 75: 15 days after the third dose on day $75)$. The $y$-axis represents the ELISA absorbance values at $492 \mathrm{~nm}$ of the sera samples diluted a ratio of 1:80. a: anti-L. amazonensis total IgG; b: anti- $L$. chagasi total IgG; c: anti-L. chagasi IgG1; d: anti-L. chagasi IgG2. "Significant differences at $P<0.05$ are indicated in comparison to WPV and Placebo groups.

with WPV presented a significant gradual increase $(P<0.05)$ of specific antibody levels during immunization when compared with baseline data (before immunization). When IgG2 was evaluated, WPV induced higher levels $(P<0.05)$ of anti-Leishmania $\operatorname{IgG} 2$ after first and third doses when compared with placebo (Fig. 3d).

\section{Discussion}

The natural history of CVL has been thoroughly demonstrated in several reports, focusing attention on the relationship between biochemical/haematological changes, histopathologic features, leukocytes immunophenotic patterns and the IgG isotype levels which were directly correlated with the clinical status of the disease and parasite load (Giunchetti et al., 2006, 2007a,b; Reis et al., 2006a,b,c). Nevertheless, few studies using accurate evaluations such as flow cytometric assays for canine leukocytes considering different anti-Leishmania vaccines have been described (Giunchetti et al., 2007c). To date, different treatment strategies have been failed to achieve consistent parasitological cure in CVL without a reliable effective therapy (Baneth and Shaw, 2002; Noli and Auxilia, 2005). In this context, a dog vaccine may be the most practical and effective method to reduce the incidence of human disease, and would permit a similar vaccine for humans (Abranches et al., 1991; Gradoni, 2001). Considering the importance of a vaccine for the control of leishmaniasis and the lack of studies on cellular and humoral events that occur during and after vaccination, the understanding of the immune response elicited by a vaccine is a prerequisite for the comprehension of the mechanisms of protection against the parasite and, therefore, the development of new and effective vaccines against this disease.
Although an increasing number of molecularly defined subunit vaccines obtained by recombinant methodologies have shown some degree of efficacy against experimental leishmaniasis (Wilson et al., 1995; Webb et al., 1996, 1998; Dole et al., 2000; Campos-Neto et al., 2001), particularly in murine model, this approach still does not reflect effective results in the dog model (Gradoni, 2001; Requena et al., 2004; Khamesipour et al., 2006). However, the semi purified FML (fucose mannose ligant) antigen associated with saponin has shown encouraging results against CVL (Silva et al., 2001; Borja-Cabrera et al., 2002). Moreover, the remarkable results obtained from the vaccination of dogs with killed parasite vaccines (Lasri et al., 1999; Mayrink et al., 1996; Panaro et al., 2001; Giunchetti et al., 2007c) showed that a successful vaccine against canine leishmaniasis is still possible.

Our results showed that immunization with WPV elicited a strong cell reactivity observed with the increase of cell proliferation after first and second doses. Thus, the ability of WPV to stimulate in vitro antigenic-specific T lymphocytes after immunization may play an important role during prime naive $\mathrm{T} \mathrm{CD} 4^{+}$lymphocytes, which are the central regulators of both humoral and cellular immune responses (Stockinger et al., 2006). Interestingly, a reduction in the amount of circulating T-lymphocytes $\mathrm{CD}^{+}$ was observed in comparison to the animals that received placebo alone, which might imply the migration of primed $\mathrm{CD}^{+}$to the lymphoid organs to mediate its effectors mechanisms of immune protection. Of note, the results obtained in all experiments using the placebo group were similar to additional control animals that received saline solution (data not shown).

The expansion in T-lymphocytes Thy- $1^{+}$in the peripheral blood after vaccination with WPV may be reflected by 
the increase of subpopulation $\mathrm{CD} 8^{+}$(after first and second immunizations; Fig. 2c), which would indicate a possible mechanism for the control of parasitism, since increased levels of T-lymphocytes $\mathrm{CD} 8^{+}$appeared as the major phenotypic feature of asymptomatic disease and dogs bearing low parasite load (Reis et al., 2006b). As noted for T CD4 ${ }^{+}$ cells, circulating B-lymphocytes CD $21^{+}$were also observed in reduced numbers in the peripheral blood. Again, it would imply a possible migration to lymphoid organs and a potential cooperative relationship with those activated $\mathrm{CD}^{+}$, resulting in the polyclonal B-lymphocytes activation. It is corroborated by the strong production of anti-Leishmania total IgG after second and third immunizations. Finally, the reduction in the expression of CD45RA by peripheral blood lymphocytes in WPV (Fig. 2e) might indicate a progressive decrease in naive canine T-lymphocytes after starting the vaccination, and therefore, likely achieving an activated status.

Immunogenicity of the WPV was also observed by the high antibody production as measured by the ELISA specifically designed to detect canine antibodies of IgG isotypes specific for the crude antigens. Since IgG1 and IgG2 responses are strictly $\mathrm{T}$ cell dependent, we used them as readouts to evaluate the overall immunogenicity of the recombinant antigens in dogs (Fujiwara et al., 2005b). In addition, for humans and mice, IgG1 and IgG2 subtypes have been traditionally used as surrogates of the $\mathrm{Th} 2$ and Th1 phenotypes of immune responses, respectively. The association between IgG subtypes and the immune response in terms of the Th1/Th2 phenotype is not so straightforward in dogs yet (Day, 2007). However, experimental evidences from published canine studies do indicate a possible association between isotype production and Th1/ Th2 shifts (Chabalgoity et al., 2001; Loukas et al., 2005; Fujiwara et al., 2006). In this way, the analysis of immunoglobulin isotypes might point out to a mixed immune response in WPV group, with initial production of $\mathrm{IgG} 2$ (after first dose) and subsequent production of IgG1 (after second dose); after third immunization, the responses of both antibodies IgG1 and $\mathrm{IgG} 2$ were presented.

Furthermore, WPV group displayed significant higher anti-Leishmania total IgG after second and third immunizations (Fig. 3a and b). Additionally, the seric reactivity using SLcA indicated antigenic recognition against $L$. chagasi, suggesting a potential use of this vaccine against the etiological agent of visceral leishmaniasis. In fact, according to Rosario et al. (2005) the antigens of L. amazonensis showed cross-reactivity with serological analysis in naturally $L$. chagasi-infected dogs, demonstrating an analogous antigenic repertory between these Leishmania species. As expected, the vaccination with WPV also induced production of antibodies that recognized SLaA, which suggests that it might be used for vaccination against American tegumentar leishmaniasis in dogs as well.

Our findings highlight on the importance in progress of vaccines using whole parasites approach, particularly in developed countries. This vaccine design has been attrac- tive in terms of cost, safety and stability when compared to purified subunits preparations or DNA vaccines which involved a sophisticated technology. The killed vaccines present a great diversity in antigenic repertory that would potentially activate a strong cellular response, mainly by T-lymphocytes, as a better approach when compared to purified subunits preparations or DNA vaccines. In this context, the present study showed that immunization with WPV induced both cellular and humoral immune responses, observed mainly as cellular reactivity (cell proliferation) and increase in T-lymphocytes $\mathrm{Thy}-1^{+}$, related particularly to the subpopulation $\mathrm{CD} 8^{+}$that support the hypothesis of the potential immunomodulation to control tissue parasitism. In addition, a higher production of anti-Leishmania total $\mathrm{IgG}$, characterized by mixed isotypes profile (IgG1 and $\operatorname{IgG} 2)$, was demonstrated. Further investigations will analyze the efficacy of the WPV in protection against the experimental challenge with $L$. chagasi.

\section{Acknowledgments}

We are grateful to Dr. Odair Genaro and Roberto Teodoro da Costa, both in memoriam, for their contribution to this work and for the research in the field of epidemiology, drugs and vaccines against canine leishmaniasis.

\section{References}

Abranches, P., Santos-Gomes, G., Rachamim, N., Campino, L., Schnur, L.F., Jaffe, C.L., 1991. An experimental model for canine visceral leishmaniasis. Parasite Immunology 13, 537-550.

Baneth, G., Shaw, S.E., 2002. Chemotherapy of canine leishmaniosis. Veterinary Parasitology 106, 315-324.

Borja-Cabrera, G.P., Correia Pontes, N.N., Silva, V.O., Paraguai de Souza, E., Santos, W.R., Gomes, E.M., Luz, K.G., Palatnik, M., Palatnik de Sousa, C.B., 2002. Long lasting protection against canine kala-azar using the FML-QuilA saponin vaccine in an endemic area of Brazil (São Gonçalo do Amarante, RN). Vaccine 20, 3277-3284.

Campos-Neto, A., Porrozzi, R., Greeson, K., Coler, R.N., Webb, J.R., Seiky, Y.A., Reed, S.G., Grimaldi, G.Jr., 2001. Protection against cutaneous leishmaniasis induced by recombinant antigens in murine and nonhuman primate models of the human disease. Infection and Immunity 69, 4103-4108.

Chabalgoity, J.A., Moreno, M., Carol, H., Dougan, G., Hormaeche, C.E., 2001. Salmonella typhimurium as a basis for a live oral Echinococcus granulosus vaccine. Vaccine 19, 460-469.

Day, M.J., 2007. Immunoglobulin G subclass distribution in canine leishmaniosis: a review and analysis of pitfalls in interpretation. Veterinary parasitology $147,2-8$.

Deane, L.M., Deane, M.P., 1962. Visceral leishmaniasis in Brazil. Geographical distribution and transmission. Revista do Instituto de Medicina Tropical de São Paulo 4, 149-212.

Dole, V.S., Raj, V.S., Ghosh, A., Madhubala, R., Myler, P.J., Stuart, K.D., 2000. Immunization with recombinant LD1 antigens protects against experimental leishmaniasis. Vaccine 15, 423-430.

Evans, T.G., Vasconcelos, I.A.B., Lima, J.W., Teixeira, J.M., McAullife, I.T., Lopes, U.G., Pearson, R.D., Vasconcelos, A.W., 1990. Canine visceral leishmaniosis in northeast Brazil: assessment of serodiagnostic methods. The American journal of Tropical Medicine and Hygiene 42, $118-123$.

Fujiwara, R.T., Bueno, L.L., Vale, A.M., França-Silva, J.C., Costa, R.T., Quetz, J.S., Machado-Coelho, G.L., Reis, A.B., Martins-Filho, O.A., 
Genaro, O., Nascimento, E., Mayrink, W., 1995. Flow cytometric assay in peripheral blood of dogs - reference values for leukocytes from Brazilian Beagles. Polish Journal of Veterinary Sciences 8, 17-22.

Fujiwara, R.T., Vale, A.M., França-Silva, J.C., Costa, R.T., Quetz, J.S., Martins-Filho, O.A., Reis, A.B., Corrêa-Oliveira, R., MachadoCoelho, G.L., Bueno, L.L., Bethony, J.M., Frank, G., Nascimento, E., Genaro, O., Mayrink, W., Reed, S., Campos-Neto, A., 2005b. Immunogenicity in dogs of three recombinant antigens (TSA, LeIF and LmSTI1) potential vaccine candidates for canine visceral leishmaniasis. Veterinary Research 36, 827-838.

Fujiwara, R.T., Loukas, A., Mendez, S., Williamson, A.L., Bueno, L.L., Wang, Y., Samuel, A., Zhan, B., Bottazzi, M.E., Hotez, P.J., Bethony, J.M., 2006. Vaccination with irradiated Ancylostoma caninum third stage larvae induces a Th2 protective response in dogs. Vaccine 24, 501-509.

Giunchetti, R.C., Mayrink, W., Genaro, O., Carneiro, C.M., CorrêaOliveira, R., Martins-Filho, O.A., Marques, M.J., Tafuri, W.L., Reis, A.B., 2006. Relationship between canine visceral leishmaniosis and the Leishmania (Leishmania) chagasi burden in dermal inflammatory foci. Journal of Comparative Pathology 135, 100-107.

Giunchetti, C., Martins-Filho, O.A., Carneiro, C.M., Mayrink, W., Marques, M.J., Tafuri, W.L., Correa-Oliveira, R., Reis, A.B., 2007a. Histopathology, parasite density and cell phenotypes of the popliteal lymph node in canine visceral leishmaniasis. Veterinary Immunology and Immunopathology. doi:10.1016/j.vetimm.2007.07.009.

Giunchetti, R.C., Mayrink, W., Carneiro, C.M., Correa-Oliveira, R., Martins-Filho, O.A., Marques, M.J., Tafuri, W.L., Reis, A.B., $2007 \mathrm{~b}$. Histopathological and immunohistochemical investigations of the hepatic compartment associated with parasitism and serum biochemical changes in canine visceral leishmaniasis. Research in Veterinary Science. doi:10.1016/j.rvsc.2007.04.020.

Giunchetti, R.C., Corrêa-Oliveira, R., Martins-Filho, O.A., TeixeiraCarvalho, A., Roatt, B.M., Aguiar-Soares, R.D.O., Vitoriano-Souza, J., Moreira, N.D., Malaquias, L.C.C., Castro, L.L.M., Lana, M., Reis, A.B., 2007c. Immunogenicity of a killed Leishmania vaccine with saponin adjuvant in dogs. Vaccine. doi:10.1016/j.vaccine.2007.08.009.

Gradoni, L., 2001. An update on antileishmanial vaccine candidates and prospects for a canine Leishmania vaccine. Veterinary Parasitology 100, 87-103.

Hommel, M., Jaffe, C.L., Travi, B., Milton, G., 1995. Experimental models for leishmaniasis and for testing anti-leishmanial vaccines. Annals of Tropical Medicine and Parasitology 89, 55-73.

Khamesipour, A., Rafati, S., Davoudi, N., Maboudi, F., Modabber, F., 2006. Leishmaniasis vaccine candidates for development: a global overview. The Indian Journal of Medical Research 123, 423-438.

Loukas, A., Bethony, J.M., Mendez, S., Fujiwara, R.T., Goud, G.N., Ranjit, N., Zhan, B., Jones, K., Bottazzi, M.E., Hotez, P.J., 2005. Vaccination with recombinant aspartic hemoglobinase reduces parasite load and blood loss after hookworm infection in dogs. PLoS medicine 2, 1008-1017.

Lasri, S., Sahibi, H., Sadak, A., Jaffe, C.L., Rhalem, A., 1999. Immune responses in vaccinated dogs with autoclaved Leishmania major promastigotes. Veterinary Research 30, 441-449.

Lemesre, J., Holzmuller, P., Cavaleyra, M., Gonçalves, R.B., Hottin, G., Papierok, G., 2005. Protection against experimental visceral leishmaniasis infection in dogs immunized with purified excreted secreted antigens of Leishmania infantum promastigotes. Vaccine 23, 28252840.

Mayrink, W., Genaro, O., Franca-Silva, J.C., da Costa, R.T., Tafuri, W.F., Toledo, V.P., da Silva, A.R., Reis, A.B., Williams, P., da Costa, P.W., 1996. Phase I and II open clinical trials of a vaccine against Leishmania chagasi infections in dogs. Memórias do Instituto Oswaldo Cruz 91, 695-697.

Molano, I., García-Alonso, M., Mirón, C., Redondo, E., Requena, J.M., Soto, M., Gómez-Nieto, C., Alonso, C., 2003. A Leishmania infantum multi-component antigenic protein mixed with live BCG confers protection to dogs experimentally infected with $L$. infantum. Veterinary Immunology and Immunopathology 92, 1-13.
Noli, C., Auxilia, S.T., 2005. Treatment of canine Old World visceral leishmaniasis: a systematic review. Veterinary Dermatology 16, 213 232.

Nunes, M.P., Jackson, J.M., Carvalho, R.W., Furtado, N.J., Coutinho, S.G., 1991. Serological survey for canine cutaneous and visceral leishmaniosis in area at risk for transmission in Rio de Janeiro where prophylactic measures had been adapted. Memórias do Instituto Oswaldo Cruz 86, 411-417.

Panaro, M.A., Acquafredda, A., Lisi, S., Lofrumento, D.D., Mitolo, V., Sisto, M., Fasanella, A., Trotta, T., Bertani, F., Consenti, B., Brandonisio, O., 2001. Nitric oxide production by macrophages of dogs vaccinated with killed Leishmania infantum promastigotes. Comparative Immunology, Microbiology and Infectious Diseases 24, 187-195.

Ravindran, R., Ali, N., 2004. Progress in vaccine research and possible effector mechanisms in visceral leishmaniasis. Current Molecular Medicine 4, 697-709.

Reis, A.B., Carneiro, C.M., Carvalho, M.G., Teixeira-Carvalho, A., Giunchetti, R.C., Mayrink, W., Genaro, O., Correa-Oliveira, O.A., Martins-Filho, O.A., 2005. Establishment of a microplate assay for fow cytometric assessment and its use for the evaluation of age-related phenotypic changes in canine whole blood leukocytes. Veterinary Immunology and Immunopathology 103, 173-185.

Reis, A.B., Martins-Filho, O.A., Teixeira-Carvalho, A., Carvalho, M.G., Mayrink, W., França-Silva, J.C., Giunchetti, R.C., Genaro, O., Corrêa-Oliveira, R., 2006a. Parasite density and impaired biochemi$\mathrm{cal} /$ hematological status are associated with severe clinical aspects of canine visceral leishmaniasis. Research in Veterinary Science 81, 68-75.

Reis, A.B., Teixeira-Carvalho, A., Giunchetti, R.C., Guerra, L.L., Carvalho, M.G., Mayrink, W., Genaro, O., Corrêa-Oliveira, R., Martins-Filho, O.A., 2006b. Phenotypic features of circulating leucocytes as immunological markers for clinical status and bone marrow parasite density in dogs naturally infected by Leishmania chagasi. Clinical and Experimental Immunology 146, 303-311.

Reis, A.B., Teixeira-Carvalho, A., Vale, A.M., Marques, M.J., Giunchetti, R.C., Mayrink, W., Guerra, L.L., Andrade, R.A., Corrêa-Oliveira, R., Martins-Filho, O.A., 2006c. Isotype patterns of immunoglobulins: hallmarks for clinical status and tissue parasite density in brazilian dogs naturally infected by Leishmania (Leishmania) chagasi. Veterinary Immunology and Immunopathology 112, 102-116.

Requena, J.M., Iborra, S., Carrión, J., Alonso, C., Soto, M., 2004. Recent advances in vaccines for leishmaniasis. Expert Opinion on Biological Therapy 4, 1505-1517.

Rosario, E.Y., Genaro, O., Franca-Silva, J.C., da Costa, R.T., Mayrink, W., Reis, A.B., Carneiro, M., 2005. Evaluation of enzyme-linked immunosorbent assay using crude Leishmania and recombinant antigens as a diagnostic marker for canine visceral leishmaniasis. Memórias do Instituto Oswaldo Cruz 2, 197-203.

Silva, V.O., Borja-Cabrera, G.P., Correia-Pontes, N.N., Paraguai de Souza, E., Luz, K.G., Palatnik, M., Palatnik de Sousa, C.B., 2001. A phase III trial of efficacy of the FML-vaccine against canine kala-azar in an endemic area of Brazil (São Gonçalo do Amaranto, RN). Vaccine 19, 1082-1092.

Stockinger, B., Bourgeois, C., Kassiotis, G., 2006. CD4 ${ }^{+}$memory T cells: functional differentiation and homeostasis. Immunological Reviews 211, 39-48.

Webb, J.R., Kaufmann, D., Campos-Neto, A., Reed, S.G., 1996. Molecular cloning of a novel protein antigen of Leishmania major that elicits a potent immune response in experimental murine leishmaniasis. Journal of Immunology 157, 5034-5041.

Webb, J.R., Campos-Neto, A., Ovendale, P.J., Martin, T.I., Stromberg, E.J., Badaró, R., Reed, S.G., 1998. Human and murine immune responses to a novel Leishmania major recombinant protein encoded by members of a multicopy gene family. Infection and Immunity 66 , 3279-3289.

Wilson, M.E., Young, B.M., Andersen, K.P., Weinstock, J.V., Metwali, A., Ali, K.M., Donelson, J.E., 1995. A recombinant Leishmania chagasi antigen that stimulates cellular immune responses in infected mice. Infection and Immunity 63, 2062-2069. 